\title{
Neue Karrieremodelle in der Inneren Medizin
}

\author{
Elisabeth Webera, Lars C. Huber ${ }^{b}$
}

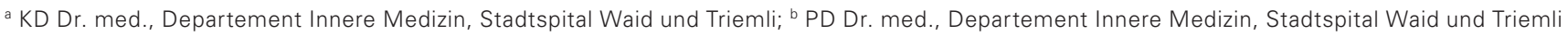

\begin{abstract}
Wir sind überzeugt, dass die unscharfe Positionierung der Inneren Medizin im Spital durch ein Vakuum an Karriereoptionen für unsere jungen allgemeininternistischen Kaderärztinnen und Kaderärzte mitbedingt ist - und vice versa. Wir setzen uns deshalb dafür ein, dass in der Inneren Medizin neue Karrieremodelle etabliert werden.
\end{abstract}

Neue Karrieremodelle für junge Ärztinnen anzubieten ist keine neue Forderung: "Establish less rigid career structures» hat Buddeberg-Fischer bereits vor beinahe 10 Jahren konkludiert [1]. Diese Aussage ist heute aktueller denn je. Sie impliziert aber auch ein Umdenken bei den Klinikleitungen gleichermassen wie bei der neuen Generation. Traditionelle Curricula im akademischen Setting und mit Spezialisierung als einziger Voraussetzung für eine Kaderposition in der Inneren Medizin sind gegenüber individuell angepassten Karrieremöglichkeiten Auslaufmodelle. Wir sind der festen Überzeugung, dass Kaderpositionen an Führungspersönlichkeiten mit fachlicher Kompetenz vergeben werden müssen - dabei sollte die Fähigkeit zur Übernahme von Leitungsfunktion gegenüber anderen Faktoren (Spezialisierung oder Forschung) im Fokus stehen. Dieses Umdenken wird von gut etablierten Vorbildern leben: an Nachwuchs in der allgemeinen Inneren Medizin fehlt es grundsätzlich nicht. Die meisten Ärzte in der Schweiz sind im Bereich der Inneren Medizin tätig (22,1\%) [2] - uns obliegt die Aufgabe, den Nachwuchs mit neuen Ideen im Spitalsystem und in der Inneren Medizin halten zu können.

\section{Entwicklung der Inneren Medizin}

Die vielschichtigen Schwierigkeiten der Inneren Medizin im Spital sind hinlänglich bekannt. Auf den kürzesten Nenner gebracht hat der immense Wissenszuwachs in den ehemaligen Subdisziplinen zu einer zunehmenden Spezialisierung und damit zur Aufsplitterung einer der grossen medizinischen Disziplinen in verschiedene, eigenständige Fachgebiete geführt [3]. Heute ist die Erlangung eines Facharzttitels in einer Spezialdisziplin auch ohne Facharzt Allgemeine Innere
Medizin möglich, und der Weg in die Spezialdisziplin führt nicht mehr in jedem Fall über eine Kaderstelle in der Inneren Medizin. Finanzielle Entwicklungen in einem Tarifsystem, welches die internistischen Leistungen nicht vernünftig abbildet und personalintensivere und spezialisierte Komplexbehandlungen fördert, haben diese Entwicklung zusätzlich beschleunigt [4]. Überspitzt gesagt haben die Zentrifugalkräfte internistische Rumpfkliniken hinterlassen, deren Ärzte sich tagsüber um die administrative Basisarbeit kümmern und nachts und am Wochenende die Frontarbeit in den Spezialkliniken, in Organzentren und auf den Notfallstationen übernehmen. $\mathrm{Zu}$ diesen Randzeiten ist der Internist weiterhin «die erste Ansprechperson, welche sich [...] umfassend, kontinuierlich und effizient um Menschen mit Gesundheitsanliegen kümmert» [5]. Gleichzeitig wird zu recht verlangt, dass der Internist Patienten mit häufigen Krankheiten und Polymorbidität ganzheitlich behandelt und sie, im Spannungsfeld mehrerer involvierter Spezialisten, vor Über- und Fehlversorgung schützt. Kurz: Für eine «wirksame, zweckmässige, wirtschaftliche» Behandlung der Patienten ist der erfahrene Internist/die erfahrene Internisitin im Spital unerlässlich.

Aber dies ist nicht umsonst zu haben: Diese Fähigkeiten erfordern nämlich in erster Linie eine integrative Syntheseleistung, aber auch Entscheidungswille unter immanentem Handlungsdruck [6], die Übernahme von Verantwortung und einen bewussten Umgang mit Unsicherheit - alles Herausforderungen, die erst mit Erfahrung bewältigt werden können. Entsprechend fällt dies den immer jünger werdenden internistischen Kaderärztinnen schwer. Die Situation wird durch häufige Fluktuationen auf der Ebene der internistischen Oberärzte zusätzlich verschärft: Die meist fehlenden 
Karriereaussichten und die Profilunschärfe des Spitalinternisten vermindern die Attraktivität und führen zu Wechseln in die Hausarztmedizin oder in eine Weiterbildung zur Spezialistin. Diese Aussage gilt gleichermassen für rein klinisch tätige Kollegen und solche, die eine akademische Laufbahn verfolgen, da die «internistische Forschung» bis auf wenige Ausnahmen immer in einem Hybridmodell mit einer anderen Disziplin stattfindet.

Die Frage nach den eigenen Karrieremöglichkeiten im Spital und der Zukunft der Inneren Medizin wird uns im Alltag von jungen begabten Internisten oft gestellt.

Die Frage nach den eigenen Karrieremöglichkeiten im Spital und der Zukunft der Inneren Medizin wird uns im Alltag von jungen begabten Internisten oft gestellt.

Die Antwort fällt auch mit neuen Modellen («Berufsbild Hospitalist») unbefriedigend aus. Solange Abgänger dieses Hospitalisten-Tracks maximal in der Funktion einer Oberärztin tätig sein können und die Frage der Zukunft der Innere Medizin im Spital nicht befriedigend beantwortet wird, fällt die Laufbahnwahl nicht zugunsten des Spitalinternisten aus. Wir sind überzeugt, dass dieser Mangel an Karriereoptionen in der Inneren Medizin einen Hauptgrund für die Schwierigkeit darstellt, unsere jungen Kollegen längerfristig für die internistische Spitalmedizin zu begeistern und im System Spital halten zu können [7].

\section{Grundproblem}

Die wenig klare Positionierung der Inneren Medizin im Spital und die daraus resultierende Unsicherheit, wie es mit der Inneren Medizin weitergeht, verschuldet das Fehlen eines eigenen Karriereprofils in der stationären Inneren Medizin - und umgekehrt. Die Erlangung eines zweiten Facharzttitels ist dabei praktisch

\section{Die Erlangung eines zweiten Facharzttitels ist praktisch zur conditio sine qua non für eine Leitungsfunktion in der Inneren Medizin geworden.}

zur conditio sine qua non für eine Leitungsfunktion in der Inneren Medizin geworden: Ob der entsprechende Arzt tatsächlich auch Führungsverantwortung wahrnimmt, ist dabei sekundär. Die Innere Medizin funktioniert so heute in erster Linie als Durchlauferhitzer mit hoher Dienstlast. Diese Aussichten sind suboptimal, um junge internistische Ärztinnen für eine Spital- karriere in der Inneren Medizin zu begeistern. Entsprechend müssen Wege gefunden werden, dies zu ändern. Im Folgenden möchten wir deshalb gerne ein einfaches Massnahmenpaket zur Diskussion stellen, das auf unsere eigene Erfahrung in der Leitung einer grossen internistischen Klinik abstellt.

\section{Massnahmen}

\section{Massnahme 1: Ausarbeitung von internistischen Oberarzt-Tracks}

Die Entwicklung und Förderung von designierten Assistententracks - wie es das Berner Curriculum für allgemeine Innere Medizin vorsieht -, welches eine "vollständige, strukturierte, [und] bis zu fünfjährige Weiterbildung zur Fachärztin» ermöglichen soll, ist zweifellos ein Schritt in die richtige Richtung [8]. Gleichzeitig wird klar, dass es unmöglich genügen kann, den $\mathrm{Zu}$ fluss an spitalinternistischen Oberärzten zu erhöhen, ohne auch den Abfluss (konkret: den Aufstieg in eine höhere Kaderposition) zu öffnen. Es ist auch illusorisch anzunehmen, dass alle Kandidaten für eine höhere Kaderposition in einem nicht-universitären Setting forschen und habilitieren werden. Dies ist auch nicht sinnvoll. Zweckmässig und zielführend ist aber eine Fortsetzung des multimodularen Aufbaus auch auf Oberarztebene: Ein Internist, der sich längerfristig in einer Leitungsfunktion in der Inneren Medizin im Spitalsetting sieht, bedarf einer anderen Betreuung als eine Oberärztin, die sich während einiger Jahre ihren internistischen Rucksack füllen will, bevor sie in eine Hausarztpraxis wechselt. Wieder anders sieht die Situation aus bei Oberärzten, welche die Innere Medizin als Zwischenstation («Warteplatz») für eine Weiterbildungsstelle zum Spezialisten sehen. Neben der unabdingbaren individuellen Karriereplanung mittels Mentoringsystem schwebt uns deshalb ein trimodales Modell als Gerüst für die grobe Planung vor. Als Diskriminatoren für die verschiedenen Modelle kommen Faktoren wie die fachliche Betreuung, das Angebot von Spezialfortbildungen, Führungskursen und Schwerpunkten in der Einsatzplanung zum Tragen (Abb. 1).

\section{Massnahme 2: Etablierung von Nischen}

Unter Nische verstehen wir die Expertise in einem integrativen medizinischen Gebiet mit Berührungspunkten zu verschiedenen Disziplinen, für welche aber keine Weiterbildung zum Spezialisten erfolgen muss. Die entsprechende Kenntnis macht den jeweiligen Kaderarzt aber in einem Gebiet zum Experten. Möglichkeiten bieten sich dazu fachlicher Art - z.B. durch Erlangen eines zusätzlichen Fähigkeitsauswei- 


\begin{tabular}{|c|c|c|c|}
\hline Track & Mentoring & Fachliche Förderung & Einsatzgebiete \\
\hline Spitalkader & $\begin{array}{l}\text { Chefarzt } \\
\text { Innere Medizin }\end{array}$ & $\begin{array}{l}\text { - „Internal Rounds“ } \\
\text { - spitalübergreifende Boards } \\
\text { zur Diskussion komplex - } \\
\text { internistischer Patienten } \\
\text { - Führungskurse } \\
\text { - Förderung einer Nische } \\
\text { - Punktionswochen } \\
\text { (strukturiertes Üben von } \\
\text { Pleura-, Aszites-, Knochen- } \\
\text { markspunktionen) } \\
\text { - Point-of-care Sonographien }\end{array}$ & $\begin{array}{l}\text { - Notfall*) } \\
\text { - Station } \\
\text { - Internistische } \\
\text { Spezialsprechstunde }\end{array}$ \\
\hline $\begin{array}{l}\text { Praxis/ } \\
\text { Hausarzt }\end{array}$ & Hausarzt & $\begin{array}{l}\text { - Psychosomatik } \\
\text { - Ambulante Medizin (Notfall) } \\
\text { - Sonographie }\end{array}$ & $\begin{array}{l}\text { - Notfallpraxis } \\
\text { - Tagesklinik }\end{array}$ \\
\hline Spezialisierung & Spezialist & $\begin{array}{l}\text { - Spezialvorträge } \\
\text { - Teilnahme an } \\
\text { Spezialrapporten }\end{array}$ & $\begin{array}{l}\text { - Notfall } \\
\text { - Station }\end{array}$ \\
\hline
\end{tabular}

*) der junge internistische Kaderarzt muss eng in die Dienste eingebunden werden. Im Verlauf kann angeboten werden, die Dienstbelastung zu reduzieren - vergleichbar mit Kollegen, die sich spezialisieren und weniger oder nicht mehr an erster „Front“ im Nacht-/ Wochenenddienst tătig sind.

Abbildung 1: Trimodales Modell (Oberarzt-Tracks).

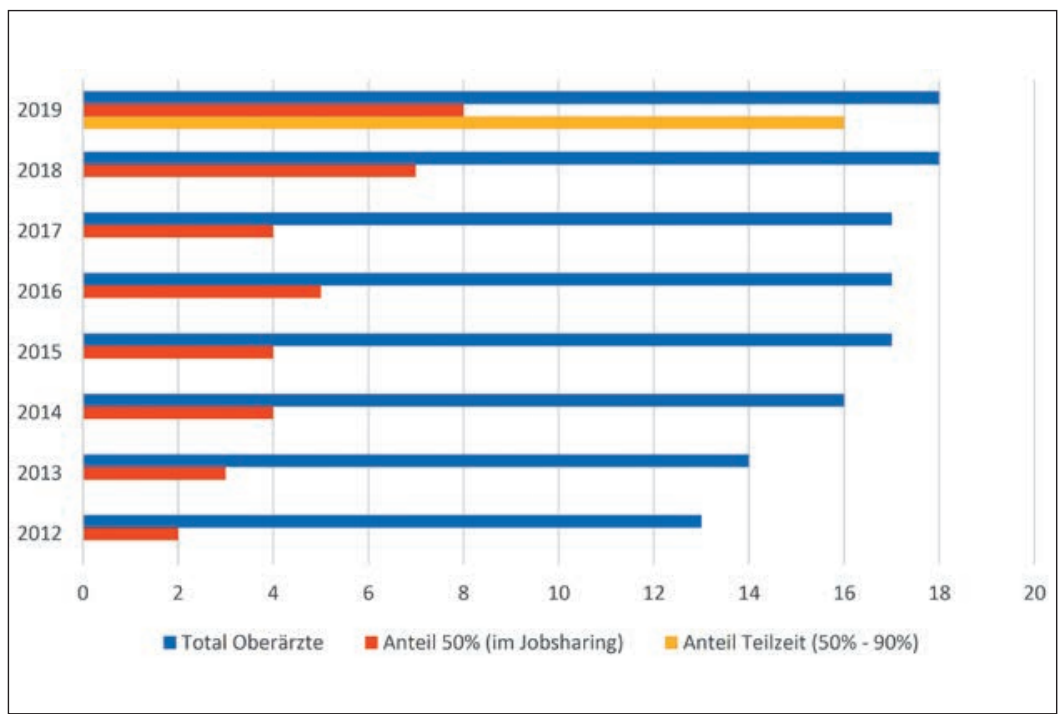

Abbildung 2: Teilzeitanteil der Oberärzte (Klinik Innere Medizin Stadtspital Triemli).

ses (Notfall, psychosomatische Medizin, Abhängigkeitserkrankungen etc.) oder die vertiefte Auseinandersetzung mit einer spezifischen Entität, die nicht

\section{Die jungen Kollegen für eine Spitalkarriere zu motivieren verlangt Teilzeitarbeit.}

einem Fachgebiet zugeordnet werden kann (Systemerkrankungen, IgG4-assoziierte Krankheiten, Sarkoidose, Amyloidose, pulmonale Hypertonie etc.). Ent- sprechende Aktivitäten können isoliert oder integrativ in Eigenregie an Schnittstellen mit einzelnen Spezialitäten aufgezogen und entwickelt werden (Stichwort: «Board»); dabei werden die Kaderärzte von der Klinikleitung (Mentor) unterstützt. Solche fachlichen Vertiefungen sind gewinnbringend für die Klinik im Alltag und ermöglichen den Kollegen die Etablierung einer persönlichen Kompetenz im fachlich doch sehr breiten Gebiet der Inneren Medizin. Daneben kann man sich auch methodisch-technische Nischen (Sonographie, Punktionen, Teaching), ökonomische Aspekte (MBA, Klinikmanagement) oder Themen aus der Medizininformatik und andere mehr vorstellen - je nach Motivation und Interesse der Kandidatin.

\section{Massnahme 3: Jobsharing}

Die jungen Kollegen für eine Spitalkarriere zu motivieren verlangt Teilzeitarbeit: Bereits 2013 waren mehr als die Hälfte der neuerworbenen Titelträger des Facharztes in Allgemeiner Innerer Medizin Frauen [9], und die Mehrheit der weiblichen Mitarbeitenden arbeitet heute in Teilzeitpensen [2]. Entsprechend müssen für alternative Arbeitspensen Möglichkeiten geschaffen werden, da wir ansonsten Gefahr laufen, diese Kolleginnen in den ambulanten Bereich zu verlieren. Ein Beispiel dazu aus unserer Klinik: Die Entwicklung von Teilzeitstellen schreitet seit 2012 rasant voran. So arbeiten aktuell von insgesamt 18 Oberärzten 16 in Teilzeit, 8 davon (44\%) im Jobsharing (Abb. 2).

Wir finden, dass diese Entwicklung als Gewinn für das Team, den klinischen Betrieb und die Patienten erkannt werden soll: Die hochmotivierten, erfahrenen und sehr gut ausgebildeten Ärztinnen bleiben so auch nach dem Mutterschaftsurlaub erhalten. Vor allem das Arbeitsmodell «Jobsharing» ermöglicht einen geregelten Wiedereinstieg in die berufliche Tätigkeit, auch im Spital. Mittelfristig werden die Stellenprozente mit dem Älterwerden der Kinder dann häufig und gerne auch wieder aufgestockt - das Jobsharing-Modell birgt so auch eine konkrete Investition in die Zukunft. Zudem scheinen die häufiger durchgeführten Übergaben auch als internes Qualitätsmerkmal auf fachlicher Ebene zu funktionieren: Die Diskussion über Beurteilung und Prozedere bei den Übergaben kann auch als kleine Intervision gesehen werden.

Explizit sollen die neuen und flexiblen Arbeitsmodelle beiden Elternteilen gleichermassen offenstehen und es ermöglichen, weiterhin im Spitalsetting tätig zu bleiben und karrieretechnisch vorwärtszukommen. Die Aufteilung von Kinderbetreuung und Arbeitstätigkeit («Dual-physicans household») ist dabei gemäss einer kürzlich im JAMA veröffentlichten Studie bei Ärztepaaren ein zunehmendes Rollenmodell [10]. 


\section{Konklusion}

Es braucht keine expliziten Karrierekurse für Internisten - weil es die Karriere nicht mehr gibt. Hingegen müssen die jungen Kollegen motiviert werden, ihren Weg gemäss ihren Interessen und Fähigkeiten zu gehen - damit und mit der Übernahme von Führungsverantwortung werden sie den Grundstein für ihren persönlichen Erfolgsweg im Spital legen. Die Innere Medizin wird dadurch auch im Spital wieder ein klares Profil mit erfahrenen Internisten erhalten. Konkret heisst das: vorleben, wie man mit Begeisterung für die Medizin - der wichtigsten Eigenschaft des Internisten [11] - die Energie und die Lust für einen langen internistischen Karriereweg im Spital aufbringen kann. Dies fordert viel ab: Geduld, Ausdauer, Frustrationstoleranz bei den jungen Kaderärzten. Das Vorleben und das Angebot entsprechender Rollenmodelle seitens der internistischen Klinikleitungen. Und das Commitment von Spitaldirektionen mit gesundheitsökonomischem Weitblick fernab von quick wins und low hanging fruits $\mathrm{zu}$ einer sichtbaren Inneren Medizin mit Zukunft, welche die gesellschaftspolitische Verantwortung im Sinne von Wirksamkeit, Zweckmässigkeit und Wirtschaftlichkeit - wie bereits in der Hausarztmedizin

Elisabeth.Weber[at]

triemli.zuerich.ch mehrfach aufgezeigt - übernehmen kann und damit ihrer Rolle als integrativer Zentrumsdisziplin auch imSpital gerecht wird.

\section{Verdankung}

Die Autoren danken PD Dr. med. Andreas D. Kistler, Klinik Innere Medizin, Kantonsspital Frauenfeld, für die kritische Durchsicht des Manuskripts.

\section{Literatur}

1 Buddeberg-Fischer B, et al. The impact of gender and parenthood on physicians' careers - professional and personal situation seven years after graduation. BMC Health Serv Res. 2010;10:40.

2 Hostettler S, Kraft E. FMH-Ärztestatistik 2018: Wenig Frauen in Kaderpositionen. Schweiz Ärzteztg. 2019;100(12):411-6.

3 Gaspoz JM. Woher sie kommt, wohin sie geht. Prim Hosp Care. 2017;17(17):323.

4 Beck T, et al. SwissDRG: Benachteiligung in der Abgeltung multimorbider Patienten. Schweiz Ärzteztg. 2018;99(12):372-7.

5 Weiterbildungsprogramm FMH AIM. 1. Januar 2011 (letzte Revision 20. Dezember 2018).

6 Maio G. Warum die gesamte Medizin von der Psychosomatik viel lernen kann. Prim Hosp Care. 2018;18(22):408-10.

7 West CP, Dupras DM. General Medicine vs Subspecialty Career Plans Among Internal Medicine Residents. JAMA. 2012;308(21) 2241-7.

8 Streit S, et al. Das Berner Curriculum für Allgemeine Innere Medizin. Prim Hosp Care. 2018;18(14):245-7.

9 Häusler N. Bundesamt für Gesundheit. Statistikbericht Humanmedizin 2013.

10 Ferrante L, Mody L. Dual-Physician Households Strategies for the 21st Century. JAMA. 2019;321(22):2161-2.

11 Salomon F. Was Internisten wissen müssen. Zeitpunkt. 2013;24-6.

\section{Das Wichtigste in Kürze}

- Die Positionierung der Inneren Medizin im Spital ist unklar; die daraus resultierende Unsicherheit, wie es mit der Inneren Medizin weitergeht, verschuldet das Fehlen eines eigenen Karriereprofils in der stationären Inneren Medizin - und umgekehrt.

- Diese Aussichten sind suboptimal, um junge internistische Ärztinnen für eine Spitalkarriere in der Inneren Medizin zu begeistern.

- Die Autoren stellen ein Massnahmenpaket zur Diskussion, das auf eigenen Erfahrungen in der Leitung einer grossen internistischen Klinik abstellt.

\section{L'essentiel en bref}

- Le positionnement de la médecine interne à l'hôpital n'est pas clair. II en résulte une incertitude sur la façon de continuer à procéder avec cette discipline, qui entraîne à son tour un manque de profils de carrière spécifiques dans la médecine interne stationnaire, et vice versa.

- Ces perspectives ne sont pas idéales pour attirer de jeunes médecins internistes vers les carrières hospitalières en médecine interne.

- Les auteurs mettent en débat une série de mesures, inspirées par leur propre expérience de direction d'un important service de médecine interne. 